\title{
Axioms which imply GCH
}

by

Jan Mycielski (Boulder, CO)

\begin{abstract}
We propose some new set-theoretic axioms which imply the generalized continuum hypothesis, and we discuss some of their consequences.
\end{abstract}

1. Introduction, motivations and summary of results. The generalized continuum hypothesis (GCH) says that $2^{\alpha}=\alpha^{+}$for all infinite cardinals $\alpha$; it is due to Cantor (for $\alpha=\aleph_{0}$ ) and to Hausdorff (for all $\alpha$ ). GCH greatly simplifies and strengthens the theory of infinite sets. The main theorems concerning GCH (all proved in ZFC) are: Cantor's inequality $2^{\alpha}>\alpha$; König's refinements $\operatorname{cf}\left(2^{\alpha}\right)>\alpha$ and $\alpha^{\operatorname{cf}(\alpha)}>\alpha$; the theorem of Silver [ $\left[\mathrm{Si}_{1}\right.$ ] that if GCH fails then the least $\alpha$ for which it fails cannot be singular of uncountable cofinality; and the theorem of Solovay [So] that $2^{\alpha}=\alpha^{+}$whenever $\alpha$ is singular, strong limit, and larger than the least strongly compact cardinal (for an alternative definition of strong compactness see $\left[\mathrm{M}_{6}\right]$ ). There are also "antitheorems": GCH cannot be disproved, and $\mathrm{CH}\left(2^{\aleph_{0}}=\aleph_{1}\right)$ cannot be proved, in ZFC $+(V=\mathrm{OD})+$ (all currently used large cardinal axioms). (Proofs of those independence results are not fully published, but they are known and the methods appear in $\left[\mathrm{Mc}, \mathrm{Me}, \mathrm{Si}_{2}\right]$. For related results and references see also $\left[\mathrm{FW}, \mathrm{LS}, \mathrm{W}_{2}\right]$, and for large cardinal axioms see $[\mathrm{Ka}, \mathrm{L}]$.)

We will propose in this paper new axioms which imply GCH and appear natural. They are intended to be additions to a canonical set theory, a theory which must appear consistent and well motivated.

The program of inventing axioms for a canonical set theory (strictly speaking a chain of such theories) must satisfy the following three intentions:

(1) The axioms should be simple and general.

(2) The universe of sets must not be enriched arbitrarily with unnatural sets or relations.

2000 Mathematics Subject Classification: Primary 03E10; Secondary 03 C62.

Key words and phrases: continuum hypothesis, axioms, models. 
(3) The universe of sets should contain all interesting mathematical objects.

In spite of the fact that (1) depends on the ambient culture, it is an important intention. For example $\mathrm{ZF}+\mathrm{AD}$ is not in the canonical set theory since the Axiom of Determinacy AD is too special, as it does not generalize to sets of higher ranks, while ZFC is in the canonical theory since the Axiom of Choice is simple and general.

In order to clarify the intention (2) we recall some examples. Urelements and non-well-founded membership relations are not needed in mathematics and for this reason the full axiom of extensionality and the axiom of regularity are in ZFC. Quine's set theory NF is not in the canonical set theory since its models (if any exist) would have to be rather artificial. We do not accept $2^{\aleph_{0}}>\aleph_{1}$ as an axiom for the canonical theory since we do not know any important or natural sets $X$ which would have to satisfy $2^{\aleph_{0}}>|X|>\aleph_{0}$. (For related remarks see the last Section 7 in this paper.)

In order to clarify (3) we recall other examples. The Axiom of Constructibility $(V=L)$ is not in the canonical set theory since $V=L$ excludes interesting objects. Already Gödel expected this to be the case, and in 1961 D. S. Scott proved that indeed $V=L$ precludes the existence of measurable cardinal numbers. Later it was shown that even $\mathbb{R} \subseteq L$ has such undesirable consequences (see e.g. [D]). The question if $V=\mathrm{OD}$ should be in the canonical theory is undecided since we may expect that some natural axiom will be invented which will imply that $\mathbb{R} \subseteq$ OD also fails (see Section 7.1).

There is no doubt that all large cardinal axioms which are used today are intended to be in the canonical theory. This is so because of their intrinsic interest and natural character, because of their consequences such as the Martin-Steel-Woodin theorem $\langle L[\mathbb{R}], \in\rangle \models \mathrm{AD}$ (for an outline of the proof see $[\mathrm{N}]$ ) which is important for real analysis, game theory and descriptive set theory (see e.g. the surveys $[\mathrm{Ma}]$ and $\left[\mathrm{M}_{2}\right]$ ), and because of the partition theory for infinite sets which they yield.

Some special axioms which imply the negation of $\mathrm{CH}$, and even $2^{\aleph_{0}}=\aleph_{2}$ and other theorems, were studied; see $\left[\mathrm{W}_{2}, \mathrm{CP}, \mathrm{CMP}, \mathrm{T}\right]$. Like AD, those axioms do not seem to generalize to sets of higher ranks. In those papers, it is not told explicitly if those axioms are intended for the canonical theory, or they are viewed only as properties of some special models of ZFC (e.g. models obtained by iterated forcing with perfect sets). I think that such intentions about new axioms should be clearly expressed since otherwise the canonical theory will be lost in a thicket of mutually inconsistent theories. \{Of course by suppressing such motivations (if they exist) one achieves a kind of security (which is not possible in physical sciences), the security of correct definitions, theorems and proofs. But I think that this is not satisfying since, as long as it appears possible, we should value the unicity of mathematics 
which is defined by a canonical (however tentative) set theory, and accept the risks of being on the wrong track or proposing inconsistent axioms. This is not to say that some special models such as $\langle L[\mathbb{R}], \in\rangle$ or $\langle L[\mathbb{R}] \cap$ $\left.V_{\omega+\omega}, \in\right\rangle$ are not interesting. Indeed these models are the most natural for the development of analysis and functional analysis of separable spaces. For related remarks see $\left[\mathrm{M}_{3}, \mathrm{M}_{4}, \mathrm{M}_{5}\right]$.

In this paper we will introduce two groups of axioms $\mathbf{A}, \mathbf{A}_{1}, \mathbf{A}_{2}, \mathbf{A}_{3}$ and $\mathbf{B}, \mathbf{B}_{0}, \mathbf{B}_{1}, \mathbf{B}_{2}$ and show (in ZFC) the following implications:
$\mathbf{A} \& \mathbf{B} \Rightarrow \mathrm{GCH}$, $V=\mathrm{OD} \Rightarrow \mathbf{A}_{1} \& \mathbf{A}_{2} \& \mathbf{A}_{3}$,
$\mathbf{A}_{1} \& \mathbf{B}_{0} \Rightarrow \mathrm{GCH}$$$
\mathbf{B} \Rightarrow \mathbf{B}_{0}
$$
$\left(\mathbf{A}_{1}\right.$ or $\mathbf{A}_{2}$ or $\left.\mathbf{A}_{3}\right) \Rightarrow \mathbf{A}$,
$\mathrm{GCH} \Rightarrow \mathbf{B}$,

as well as some other consequences of these axioms. The axioms $\mathbf{B}_{1}$ and $\mathbf{B}_{2}$ stand apart since their consistency (in ZFC) is not known. All the other axioms are consequences of GCH \& $V=\mathrm{OD}$, and they are intended for the canonical set theory. Perhaps $\mathbf{B}_{1}$ and $\mathbf{B}_{2}$ (if they are consistent) also deserve to be added there.

All the proofs in this paper will be very simple. The main points are the statements of the axioms and the arguments showing their natural character. Of course this paper would loose all its motivation if $V=$ OD was accepted into the canonical set theory since in that case all our axioms (except $\mathbf{B}_{1}$ and $\mathbf{B}_{2}$ ) would reduce to GCH.

A. Enayat and W. H. Woodin have proved some consequences of those axioms (see Section 6). In particular Woodin proved that a certain variant of $\mathbf{B}_{1}$ is inconsistent with $\left(\mathbf{A}_{2}\right.$ or $\left.\mathbf{A}_{3}\right)$. Originally I thought that that variant is important, and so his theorem influenced the shape of this paper in a significant way.

I am also indebted to Andreas Blass, Ali Enayat and Benedikt Löwe for stimulating correspondence and conversations in earlier stages of this work, and to Richard Laver for information about many things and especially those partly published independence and consistency proofs.

2. The simplest new axioms A and B. We will use the following standard notations and conventions: $\alpha$ will always denote infinite cardinal numbers, $\alpha^{+}$the successor cardinal of $\alpha$, and $\operatorname{cf}(\alpha)$ the least ordinal cofinal with $\alpha$. All cardinals are identified with initial ordinals, and all ordinals are sets of all smaller ordinals. For any sets $X$ and $Y,|X|$ denotes the cardinality of $X, P(X)$ the powerset of $X,{ }^{X} Y$ the set of functions $f: X \rightarrow Y$. Ord denotes the class of ordinal numbers and $V_{\xi}=\bigcup_{\eta<\xi} P\left(V_{\eta}\right)$ for every $\xi \in$ Ord. OD denotes the class of ordinal-definable sets and HOD the class of hereditarily ordinal-definable sets (see Myhill and Scott [MS]). $L$ denotes the class of Gödel's constructible sets and $L[X]$ the class of constructible 
sets with parameters in $X$. Recall that $L[X]$ is always a model of ZF but not always of ZFC. Finally $\xi+\eta$ will denote ordinal addition.

Our simplest axioms which imply GCH are the following:

A. For every $\alpha$ we have $|P(\alpha) \cap \mathrm{OD}|>\alpha$.

B. For every $\alpha$ and every set $X \subseteq P(\alpha)$ such that $|X|>\alpha$ and $X \in \mathrm{OD}$ we have $|X|=2^{\alpha}$.

\section{Theorem 1. A \& $\mathbf{B} \Rightarrow$ GCH.}

Proof. Let $X=P(\alpha) \cap$ OD. Thus $X$ has a definable well-ordering (see [MS]). By A, $X$ has an initial segment $X_{0}$ of type $\alpha^{+}$. Of course $X_{0} \in$ OD. Hence, by $\mathbf{B}, \alpha^{+}=\left|X_{0}\right|=2^{\alpha}$.

It may be worthwhile to point out that $\mathbf{A}$ is equivalent to the statement: for every $\alpha$ there exists an ordinal-definable injection $f: \alpha^{+} \rightarrow P(\alpha)$.

Now let us argue that $\mathbf{A}$ and $\mathbf{B}$ should be included in the canonical set theory. We have four arguments in favor of $\mathbf{A}$ and one in favor of $\mathbf{B}$.

1. Consider first the case $\alpha=\aleph_{0}$ of $\mathbf{A}$. Assume to the contrary that $|P(\omega) \cap \mathrm{OD}|=\aleph_{0}$. This expresses a kind of independence between $P(\omega)$ and OD. Such an independence suggests the logical independence of the continuum hypothesis $(\mathrm{CH})$. Thus, if we wish at all to decide $\mathrm{CH}$, it is more natural to assume $|P(\omega) \cap \mathrm{OD}|>\aleph_{0}$ than its negation. By analogy we think that $\mathbf{A}$ is also natural for all $\alpha$.

2. Again start with $\alpha=\aleph_{0}$. A theorem of Myhill and Scott [MS] tells us that

$$
|P(\omega) \cap \mathrm{OD}|=\left|\left\{\mathrm{Th}\left\langle V_{\xi}, \in\right\rangle: \xi \in \mathrm{Ord}\right\}\right| .
$$

Now, the language of mathematics is decribed mathematically as the firstorder language of $\mathrm{ZF}$ (or the Hilbert $\varepsilon$-extension of this language). Hence $|P(\omega) \cap \mathrm{OD}|=\aleph_{0}$ tells us that we can distinguish only $\aleph_{0}$ models $\left\langle V_{\xi}, \in\right\rangle$ in this language. This is a pessimistic assumption about the expressive power of mathematics. Thus again $|P(\omega) \cap \mathrm{OD}|>\aleph_{0}$ seems more natural. And we have the following generalization of the theorem of Myhill and Scott to all cardinals $\alpha$ :

Theorem 2. $|P(\alpha) \cap \mathrm{OD}|=\left|\left\{\operatorname{Th}\left(\left\langle V_{\alpha+\xi}, \in, \chi\right\rangle_{\chi<\alpha}\right): \xi \in \mathrm{Ord}\right\}\right|$.

Proof. Each theory $T_{\xi}=\operatorname{Th}\left(\left\langle V_{\alpha+\xi}, \in, \chi\right\rangle_{\chi<\alpha}\right)$ is in OD and the language of $T_{\xi}$ is of power $\alpha$. Hence there are at most $|P(\alpha) \cap \mathrm{OD}|$ theories $T_{\xi}$.

To show the converse inequality notice first that for each $\alpha$ there are infinitely many theories $T_{\xi}$ (e.g. those with $\xi<\omega$ are different from each other). Now we will use the fact that, given any set $X \in \mathrm{OD}$, the extent of $X$ is definable in some model $\left\langle V_{\alpha+\xi}, \in\right\rangle$ by some unary formula $\varphi$ of the language of ZF. Thus, for $X \subseteq \alpha$ and for such $\xi$ and $\varphi$, the set of sentences

$$
\left\{\varphi\left(c_{\chi}\right): \chi \in X\right\} \cup\left\{\neg \varphi\left(c_{\chi}\right): \chi \in \alpha-X\right\},
$$


where $c_{\chi}$ is the name of $\chi$ in $T_{\xi}$, is a subset of $T_{\xi}$. But there are only $\aleph_{0}$ possible formulas $\varphi$. So for all infinite $\alpha$ there must be at least $|P(\alpha) \cap \mathrm{OD}|$ distinct theories $T_{\xi}$.

Thus, by Theorem 2 and by analogy with the case $\alpha=\aleph_{0}$ which was discussed prior to Theorem 2, the full axiom $\mathbf{A}$ is also natural.

3. Our third and fourth arguments supporting $\mathbf{A}$ also support stronger axioms $\mathbf{A}_{2}$ and $\mathbf{A}_{3}$. These arguments will be formulated in Section 5, and they are perhaps more convincing than the above ones.

4. The reasons for accepting $\mathbf{B}$ are not as convincing as those supporting $\mathbf{A}$ or its variants $\mathbf{A}_{1}, \mathbf{A}_{2}$ and $\mathbf{A}_{3}$ (see below). We can only say that $\mathbf{B}$ is similar to the theorem (of an appropriate strengthening of ZFC) that all uncountable sets of reals of the class $L[\mathbb{R}]$ have perfect subsets. The latter may suggest propositions stronger than $\mathbf{B}$, but some such propositions will be disproved in Sections 4 and 6 .

In view of the less motivated status of $\mathbf{B}$, a weaker version $\mathbf{B}_{0}$ of $\mathbf{B}$ which may be more natural will be proposed in the next Section 3 .

Notice that $\mathbf{A}$ follows from $V=\mathrm{OD}$, and $\mathbf{B}$ follows from GCH. So there is no problem with the consistency of $\mathbf{A} \& \mathbf{B}$. Moreover, $\mathrm{ZFC}+(V=\mathrm{OD})+$ $\mathrm{GCH}+$ (any currently used large cardinal axiom) is consistent, assuming that ZFC + (that large cardinal axiom) is consistent. \{The proofs of those theorems are not fully published but they are known and based on methods which appear in four papers [LS, Mc, Me, $\left.\left.\mathrm{Si}_{2}\right].\right\}$

3. The variants $\mathbf{A}_{1}$ and $\mathbf{B}_{0}$. We will now state a strengthening of $\mathbf{A}$ and a weakening of $\mathbf{B}$ which together still yield GCH.

$\mathbf{A}_{1}$. For every $\alpha$ we have $\left.\right|^{\operatorname{cf}(\alpha)} \alpha \cap \mathrm{OD} \mid>\alpha$. $\alpha^{\mathrm{cf}(\alpha)}$.

$\mathbf{B}_{0}$. For all $\alpha$ and all $X \subseteq{ }^{\operatorname{cf}(\alpha)} \alpha$, if $|X|>\alpha$ and $X \in \mathrm{OD}$, then $|X|=$

We have $\mathbf{A}_{1} \Rightarrow \mathbf{A}$ since there are ordinal-definable injections ${ }^{\mathrm{cf}(\alpha)} \alpha \rightarrow$ $\alpha \times \alpha_{2} \rightarrow P(\alpha)$.

We have $\mathbf{B} \Rightarrow \mathbf{B}_{0}$ since these injections, the statement $\mathbf{B}$, and the assumption of $\mathbf{B}_{0}$ yield $|X|=2^{\alpha}$ and $2^{\alpha}=\alpha^{\operatorname{cf}(\alpha)}$.

Theorem 3. $\mathbf{A}_{1} \& \mathbf{B}_{0} \Rightarrow \mathrm{GCH}$.

Proof. We argue by induction. Suppose that $\beta^{+}=2^{\beta}$ for all cardinals $\beta<\alpha$. Then by cardinal arithmetic $\alpha^{\mathrm{cf}(\alpha)}=2^{\alpha}$. By $\mathbf{A}_{1}$ the set ${ }^{\operatorname{cf}(\alpha)} \alpha \cap$ OD has an ordinal-definable subset $S$ of power $\alpha^{+}$. Thus, by $\mathbf{B}_{0},|S|=\alpha^{\mathrm{cf}(\alpha)}$. Hence $\alpha^{+}=2^{\alpha}$.

If $\mathbf{B}$ appears too strong (for singular $\alpha$ ) then perhaps $\mathbf{B}_{0}$ is more acceptable (see also the next section). 
Concerning consistency, once again $V=\mathrm{OD} \Rightarrow \mathbf{A}_{1}$, and hence there is no problem with the consistency of $\mathbf{A}_{1} \& \mathbf{B}$. To me, $\mathbf{A}_{1}$ appears almost as natural as $\mathbf{A}$.

4. The axioms $\mathbf{B}_{1}$ and $\mathbf{B}_{2}$. In this section we state a variant $\mathbf{B}_{1}$ of $\mathbf{B}_{0}$ which is suggested by the perfect set property and another candidate axiom $\mathbf{B}_{2}$ related to the property of Baire.

Let us order ${ }^{\alpha} 2$ and ${ }^{\operatorname{cf}(\alpha)} \alpha$ in the lexicographic way.

$\mathbf{B}_{1}$. For all $\alpha$ and all $X \subseteq{ }^{\operatorname{cf}(\alpha)} \alpha$, if $|X|>\alpha$ and $X \in L\left[{ }^{\operatorname{cf}(\alpha)} \alpha\right]$, then $X$ has a subset which is order-isomorphic to ${ }^{\operatorname{cf}(\alpha)} \alpha$.

It seems that $\mathbf{B}_{1}$ could be useful if we wanted to generalize descriptive set theory to higher cardinal numbers replacing the Baire space ${ }^{\omega} \omega$ by the space ${ }^{\operatorname{cf}(\alpha)} \alpha$ with its order topology. But I do not know if ZFC $+\mathbf{B}_{1}$ is consistent. We add five remarks:

1. $\mathbf{B}_{1}$ for $\alpha=\aleph_{0}$ is equivalent to the perfect set property for the class $L[\mathbb{R}]$, i.e., the claim that all uncountable sets of reals of that class have perfect subsets. (This equivalence follows easily from the fact that a subset of the irrational numbers is order-isomorphic to the set of irrationals iff it is the image of a strictly increasing function and the fact that such a function can have at most $\aleph_{0}$ points of discontinuity.)

2 . The set ${ }^{\operatorname{cf}(\alpha)} \alpha$ in the statement of $\mathbf{B}_{1}$ cannot be replaced by the set ${ }^{\alpha} 2$. Indeed, if $\alpha=\aleph_{\omega}$, we can define a set $D \subseteq{ }^{\alpha} 2,|D|>\alpha$, and $D \in L\left[{ }^{\alpha} 2\right]$, such that $D$ has no subsets order-isomorphic to ${ }^{\alpha} 2$ nor even to $\omega_{1}$. This definition is the following: We consider first the partition $\omega_{\omega}=P_{0} \cup P_{1} \cup \ldots$, where $P_{n}$ is of order type $\omega_{n}$, and $x<y$ whenever $x \in P_{i}, y \in P_{j}$ and $i<j$. Then $D$ is defined as the set of functions $f: \omega_{\omega} \rightarrow\{0,1\}$ such that $\left|f^{-1}(1) \cap P_{n}\right|=1$ for all $n<\omega$. Thus $D \in L\left[{ }^{\alpha} 2\right]$ and $D \in \mathrm{OD}$. It is clear that the order type of $D$ is $\widetilde{\omega}_{0} \times \widetilde{\omega}_{1} \times \ldots$, where $\widetilde{\omega}_{n}$ denotes the converse of $\omega_{n}$ and the product is ordered lexicographically. Let us identify $D$ with $\widetilde{\omega}_{0} \times \widetilde{\omega}_{1} \times \widetilde{\omega}_{2} \times \ldots$ By König's inequality $|D|>\aleph_{\omega}$. Now suppose to the contrary that $D$ has a subset $E$ of order type $\omega_{1}$. Let $E_{n}$ denote the projection of $E$ into $\widetilde{\omega}_{n}$. We claim that some $E_{n}$ must be infinite. Indeed if all were finite then $E_{0} \times E_{1} \times \ldots$ would be order-isomorphic to the Cantor set ${ }^{\omega} 2$. But ${ }^{\omega} 2$ has no subsets of order type $\omega_{1}$. Then let $n_{0}$ be the least integer such that $E_{n_{0}}$ is infinite. Let $F$ be the image of the projection of $E$ into $\widetilde{\omega}_{0} \times \widetilde{\omega}_{1} \times \ldots \times \widetilde{\omega}_{n_{0}-1}$. Thus $F$ is finite, and there exists a point $p \in F$ such that the cylinder over $p$ intersected with $E$ projects to an infinite subset of $E_{n_{0}}$. Since $E_{n_{0}} \subseteq \widetilde{\omega}_{n_{0}}$, and the product is ordered lexicographically, $E$ is not well ordered. So again $E$ is not of type $\omega_{1}$.

3. In Section 6 we will state a theorem of $\mathrm{H}$. Woodin which implies that the class $L\left[{ }^{\operatorname{cf}(\alpha)} \alpha\right]$ in the statement of $\mathbf{B}_{1}$ cannot be replaced by OD, 
without violating another axiom $\mathbf{A}_{2}$ introduced there. Thus we think that such a modification of $\mathbf{B}_{1}$ cannot be added to the canonical set theory. At this point in time only $\mathbf{B}_{1}$ itself has a chance.

4. The connection of $\mathbf{B}_{1}$ with the perfect set property suggests seeking some general version of the Axiom of Determinacy (AD) for all cardinal numbers. But we do not know any such version. AD does not generalize to binary games of perfect information of length $\omega_{1}+\omega$, even when the set $X \subseteq \omega_{1}+\omega_{2}$ defining the game belongs to $\mathrm{OD}$ and to $L\left[P\left(\omega_{1}\right)\right]$. The following example of a nondetermined game of that kind is due to F. Galvin (see [St]): Player I must code in his first $\omega_{1}$ moves (in some natural way) an uncountable set $Y \subseteq{ }^{\omega} 2$ (otherwise he loses). Then, for the remaining $\omega$ moves they play the perfect set game of Morton Davis defined by $Y$, that is, II has a winning strategy iff $Y$ has a perfect subset (see e.g. $\left.\left[\mathrm{M}_{2}\right]\right)$. It follows that the whole game (of length $\omega_{1}+\omega$ ) is not determined, but it is fully defined and hence it is in OD and also in the class $L\left[P\left(\omega_{1}\right)\right]$.

5 . However, it is easy to generalize the perfect set game to the case of any regular cardinal $\alpha$ and any set $X \subseteq{ }^{\alpha} 2$ such that the resulting game has the following properties: I has a winning strategy iff $X$ has a subset lexicographic order-isomorphic to ${ }^{\alpha} 2$, and II has a winning strategy iff $|X| \leq \alpha$. This generalizes the theorem of Morton Davis from $\aleph_{0}$ to all regular $\alpha$. However, I do not know of any such generalization for singular cardinals $\alpha$.

In order to state $\mathbf{B}_{2}$ we need the following definitions. A subset $X$ of ${ }^{\operatorname{cf}(\alpha)} \alpha$ will be called $\alpha$-meager iff it is a union of at most $\operatorname{cf}(\alpha)$ nowhere dense sets in the lexicographic order topology. And $X$ will be said to have the $\alpha$-Baire property iff it is open modulo $\alpha$-meager.

\section{$\mathbf{B}_{2}$. If $X \subseteq{ }^{\mathrm{cf}(\alpha)} \alpha$ and $\left.X \in L{ }^{[\mathrm{cf}(\alpha)} \alpha\right]$, then $X$ has the $\alpha$-Baire property.}

Our former comment which follows $\mathbf{B}_{1}$ applies also to $\mathbf{B}_{2}$. And we add that it is easy to define a Banach-Mazur game of length $\operatorname{cf}(\alpha)$ such that $X \subseteq{ }^{c f(\alpha)} \alpha$ is a complement of an $\alpha$-meager set iff player II has a winning strategy.

5. Model-theoretic considerations. In this section we will show some model-theoretic theorems which will be used in the next section as suggestions for our strongest axioms $\mathbf{A}_{2}$ and $\mathbf{A}_{3}$.

Let $T$ be a countable consistent first-order theory in a language $L$ and $\delta(x)$ a unary formula in $L$.

We will say that $\delta$ is weakly well ordered iff there exists a binary formula $x<y$ in $L$ satisfying the following conditions:

(1) $T$ proves $x<y \rightarrow \delta(x) \& \delta(y)$.

(2) $T$ proves that $<$ is a linear ordering of the class defined by $\delta$. 
(3) For every unary formula $\varphi(x)$ in $L, T$ proves the implication: if $\exists x[\delta(x) \& \varphi(x)]$ then there exists a <-least element in the class defined by $\delta(x) \& \varphi(x)$.

[Sometimes it is the case that $\delta$ is strongly well-ordered, in the sense that additional free variables are allowed in $\varphi$. But this concept will not be needed in this paper.]

The work of Paris $[\mathrm{P}]$ suggests the following theorem.

THEOREM 4. There exists a model $M$ of $T$ such that for every $\delta$ weakly well-ordered by $T$ all the elements of $M$ satisfying $\delta$ are definable in $M$ by some unary formulas of $L$.

Proof. For every $\delta$ weakly well-ordered by $T$ we consider the type $\Gamma_{\delta}$ consisting of all unary formulas $\gamma_{\varphi}(x)$ of the form

$$
\gamma_{\varphi}(x)=[\delta(x) \& \neg(x \text { is the <-least element satisfying } \varphi(x))],
$$

where $\varphi$ is any unary formula in $L$.

If $a$ is an element of a model $N$ of $T$ such that $a$ satisfies $\delta(x)$ and $a$ omits the type $\Gamma_{\delta}$, then of course $a$ is defined in $N$ by one of the formulas $\neg \gamma_{\varphi}(x)$. Therefore, by the Omitting Types Theorem, in order to prove Theorem 4 it suffices to show that each of the types $\Gamma_{\delta}$ can be locally omitted, i.e., that the following is true:

For every unary formula $\psi$ in $L$, if

$$
T \text { proves } \psi(x) \rightarrow \gamma_{\varphi}(x)
$$

for all $\gamma_{\varphi} \in \Gamma_{\delta}$, then $T$ proves $\neg \psi(x)$.

Indeed, for such $\psi, T$ proves $\psi(x) \rightarrow \gamma_{\psi}(x)$. Hence by (3), $T$ proves $\neg \psi(x)$.

Consider the set-theoretic formula

L:

$$
\forall \alpha\left[x, y \in V_{\alpha} \rightarrow\left\langle V_{\alpha}, \in, x\right\rangle \equiv\left\langle V_{\alpha}, \in, y\right\rangle\right] \rightarrow x=y,
$$

where $\equiv$ denotes elementary equivalence of models.

THEOREM 5. If $T$ is a complete consistent extension of $\mathrm{ZF}$, then $T$ proves $\mathbf{L}$ iff $T$ has a model $M$ which satisfies the Leibniz definition of equality

$\mathbf{L}^{*}$ :

$$
\forall \varphi[\varphi(x) \leftrightarrow \varphi(y)] \rightarrow x=y,
$$

where $\varphi$ runs over unary formulas in the language of $\mathrm{ZF}$. Moreover if such a model $M$ exists it can be such that all the ordinals of $M$ are definable in $M$.

Proof. This theorem was already proved as Theorem 5 in $\left[\mathrm{M}_{1}\right]$. This proof is similar to that of Theorem 4. It is enough to omit two types: the 
unary type $\Gamma_{\delta_{0}}$, which is defined as in the previous proof with $\delta_{0}(x)$ defining the class of ordinal numbers, and the binary type of formulas of the form

$$
(\varphi(x) \leftrightarrow \varphi(y)) \& x \neq y,
$$

for all unary formulas $\varphi$.

REMARK. It is easy to see that every model of ZF which satisfies $\mathbf{L}^{*}$ satisfies also $\mathbf{L}$.

Let $M=\langle A, E\rangle$, where $E \subseteq A^{2}$, be a model which satisfies the axiom of extensionality, i.e.,

$$
\forall z[z E x \leftrightarrow z E y] \rightarrow x=y,
$$

and is well-founded, i.e., there exists no infinite sequence $a_{1}, a_{2}, \ldots$ of elements of $A$ such that

$$
a_{2} E a_{1}, \quad a_{3} E a_{2}, \quad a_{4} E a_{3}, \quad \ldots
$$

Let $S \subseteq A$ be an $E$-transitive set, i.e.,

$$
a E b \& b \in S \rightarrow a \in S .
$$

We have the following refinement of Mostowski's Lemma:

Theorem 6. If $f: S \rightarrow V_{\alpha}$ is an isomorphic imbedding, i.e., for $a, b \in S$ we have

$$
a E b \leftrightarrow f(a) \in f(b),
$$

then there exists some $\beta \geq \alpha$ and an extension $f^{*}: A \rightarrow V_{\beta}$ of $f$ such that $f^{*}$ is an isomorphic embedding of $M$ into $\left\langle V_{\beta}, \in\right\rangle$ and such that, for all $b \in A-S, f^{*}(b)=\left\{f^{*}(a): a E b\right\}$. Moreover such an extension $f^{*}$ is unique.

Proof. If such an $f^{*}$ exists then it must satisfy

$$
f^{*}(x)= \begin{cases}f(x) & \text { if } x \in S, \\ \left\{f^{*}(y): y E x\right\} & \text { if } x \notin S .\end{cases}
$$

Since $E$ is well-founded this is a correct recursive definition of a function $f^{*}: A \rightarrow V_{\beta}$, where $\beta \geq \alpha$. The unicity of $f^{*}$ follows. It remains to show that $f^{*}$ is an isomorphism. By the definition (4) it is clear that if $a, b \in S$ or if $b \notin S$ then

$$
a E b \leftrightarrow f^{*}(a) \in f^{*}(b) .
$$

It remains to show that (5) holds also in the case $a \notin S$ and $b \in S$. By the $E$-transitivity of $S$, in this case we have not- $(a E b)$. So it remains to show that $f^{*}(a) \notin f^{*}(b)$.

Let $\varrho_{1}: A \rightarrow$ Ord and $\varrho_{2}: V_{\beta} \rightarrow$ Ord be ranking functions which are defined as follows:

$$
\varrho_{1}(x)= \begin{cases}0 & \text { if } x \in S, \\ \bigcup\left\{\varrho_{1}(y)+1: y E x\right\} & \text { if } x \in A-S,\end{cases}
$$


and

$$
\varrho_{2}(x)= \begin{cases}0 & \text { if } x \in \operatorname{Tr}(f[S]), \\ \bigcup\left\{\varrho_{2}(y)+1: y \in x\right\} & \text { if } x \in V_{\beta}-\operatorname{Tr}(f[S]),\end{cases}
$$

where $\operatorname{Tr}(z)=z \cup \bigcup(z) \cup \bigcup \bigcup(z) \cup \ldots$

It is easy to show by induction relative to $E$ that, for all $x \in A$,

$$
\varrho_{2}\left(f^{*}(x)\right)=\varrho_{1}(x) .
$$

Also, if $x \in y$ then $\varrho_{2}(x) \leq \varrho_{2}(y)$, and $\varrho_{1}(x)>0$ for all $x \in A-S$. Then, since in our case $a \notin S$ and $b \in S$, we have $\varrho_{1}(a)>\varrho_{1}(b)$, and $\varrho_{2}\left(f^{*}(a)\right)>\varrho_{2}\left(f^{*}(b)\right)$. Thus $f^{*}(a) \notin f^{*}(b)$ follows.

THEOREM 7. If $T$ is a consistent complete extension of ZF then $T$ has a model $M$ such that all the ordinals of $M$ are definable in $M$ by unary formulas. If $T$ has a well-founded model then $M$ is also well-founded. If $T$ has a model of the form $\left\langle V_{\xi}, \in\right\rangle$ then $M$ is isomorphic to a submodel $M^{*}$ of $\left\langle V_{\xi}, \in\right\rangle$ such that each formula defining an ordinal in $\left\langle V_{\xi}, \in\right\rangle$ defines the same ordinal in $M^{*}$. If $\mathbf{L}$ is a theorem of $T$ and $\left\langle V_{\xi}, \in\right\rangle$ satisfies $T$, then $M^{*}$ can be required to satisfy the Leibniz principle $\mathbf{L}^{*}$.

This theorem follows immediately from the previous theorems of this section. Of course the isomorphism of $M$ and $M^{*}$ is given by Theorem 6 as an extension of the isomorphism of the set of ordinals of $M$ and the set of definable ordinals of $\left\langle V_{\xi}, \in\right\rangle$ given by their definitions.

Problem. We do not know if any $\left\langle V_{\xi}, \in\right\rangle$ with $\xi \geq \omega_{1}$ has an elementary submodel such that all its ordinals are definable.

6. The axioms $\mathbf{A}_{2}$ and $\mathbf{A}_{3}$. Here we will state our second and third refinements of $\mathbf{A}$, arguments supporting those refinements, and some consequences. $\mathbf{A}_{2}$ will strengthen the following well known theorem of ZF: If $\Phi$ is a finite set of ordinal numbers, then there exists a $\xi$ such that all ordinals of $\Phi$ are definable in $\left\langle V_{\xi}, \in\right\rangle$.

$\mathbf{A}_{2}$. For every $\alpha$ and every set $S$ of ordinal numbers such that $|S| \leq \alpha$, there exists an ordinal $\xi$ such that $S \subseteq \alpha+\xi$ and all the ordinals of $S$ are definable in the structure $\left\langle V_{\alpha+\xi}, \in, \eta\right\rangle_{\eta<\alpha}$.

We feel that the assumption that such a $\xi$ exists is similar to the assumptions that such and such large cardinals exist. The axiom $\mathbf{A}_{2}$ is intended for the canonical set theory, and we argue for it as follows. Theorem 7 suggests that instead of imagining Cantor's universe we can imagine a very saturated model $M^{*}$ as in that theorem. This in turn suggests the axiom $\mathbf{A}_{2}$ for $\alpha=\aleph_{0}$. (In fact $\mathbf{A}_{2}$ puts into the theory as much of the property of definability of ordinals as possible.) For larger $\alpha$ we argue by analogy. 
If the axiom $\mathbf{A}_{2}$ is too optimistic, we can restrict it to the case $S \in \alpha^{+}$.

$\left[\mathbf{A}_{2}\right.$ for the case $\alpha=\aleph_{0}$ was already formulated in $\left[\mathrm{M}_{1}\right]$ and a similar supporting argument was given there.]

Of course if $V=$ OD then, since $|S| \leq \alpha$, there exists an ordinal-definable injection of $S$ into $\alpha$. Hence $V=\mathrm{OD} \Rightarrow \mathbf{A}_{2}$.

We do not know if $\mathbf{A}_{2} \Rightarrow \mathbf{A}_{1}$. Let us show that $\mathbf{A}_{2} \Rightarrow \mathbf{A}$. Since for every $\xi$ there are at most $\alpha$ definitions in the language of the model $\left\langle V_{\alpha+\xi}, \in, \eta\right\rangle_{\eta<\alpha}$, it follows that $\mathbf{A}_{2}$ restricted to $S \in \alpha^{+}$implies that there are at least $\alpha^{+}$ theories of the form $\operatorname{Th}\left(\left\langle V_{\alpha+\xi}, \in, \eta\right\rangle_{\eta<\alpha}\right)$. Of course this implies $\mathbf{A}$.

$\mathbf{A}_{3}$. For every $\alpha$ and every set $S$ such that $|S| \leq 2^{\alpha}$ there exists an ordinal $\xi$ such that $S \subseteq V_{\alpha+\xi}$ and for all $a, b \in S, a \neq b$ we have

$$
\operatorname{Th}\left(\left\langle V_{\alpha+\xi}, \in, \eta, a\right\rangle_{\eta<\alpha}\right) \neq \operatorname{Th}\left(\left\langle V_{\alpha+\xi}, \in, \eta, b\right\rangle_{\eta<\alpha}\right),
$$

in other words $\xi$ is such that unary formulas of first-order logic separate the points of $S$ in the model $\left\langle V_{\alpha+\xi}, \in, \eta\right\rangle_{\eta<\alpha}$.

Again we feel that the assumption that such a $\xi$ exists is similar to the assumptions that such and such large cardinals exist. But $\mathbf{A}_{3}$ is intended for the canonical set theory. The argument supporting $\mathbf{A}_{3}$ is similar to that supporting $\mathbf{A}_{2}$. First Ockham's principle of economy of concepts suggests that $\mathbf{L}^{*}$ is a natural principle and hence $\mathbf{L}$ should be accepted as an axiom of the canonical set theory. Then Theorems 5 and 7 suggest that instead of imagining Cantor's universe we can imagine a very saturated model $M^{*}$ satisfying $\mathbf{L}^{*}$. (Of course $\mathbf{A}_{3}$ puts into the theory as much of the property $\mathbf{L}^{*}$ as possible.) This suggests the axiom $\mathbf{A}_{3}$ for $\alpha=\aleph_{0}$. For larger $\alpha$ we argue by analogy.

We note that, like $\mathbf{L}^{*}, \mathbf{A}_{3}$ also yields $\mathbf{L}$.

If $\mathbf{A}_{3}$ is too optimistic, we can restrict it to the case $S=\alpha^{+}$.

$\left[\mathbf{A}_{3}\right.$ for $\alpha=\aleph_{0}$ was already formulated in $\left[\mathrm{M}_{1}\right]$ and some parts of the above supporting argument were given there.]

Again we do not know if $\mathbf{A}_{3} \Rightarrow \mathbf{A}_{1}$, and again $\mathbf{A}_{3} \Rightarrow \mathbf{A}$. To check the latter it suffices to apply the case $S=\alpha^{+}$of $\mathbf{A}_{3}$. Indeed this case implies that for some $\xi$ there are at least $\alpha^{+}$distinct theories of the form $\operatorname{Th}\left(\left\langle V_{\alpha+\xi}, \in, \eta, \gamma\right\rangle_{\eta<\alpha}\right)$, where $\gamma<\alpha^{+}$, and $\mathbf{A}$ follows.

Of course if $V=$ OD then, since $|S| \leq 2^{\alpha}$, there exists an ordinaldefinable injection of $S$ into ${ }^{\alpha}$ 2. Hence it follows easily that $V=\mathrm{OD} \Rightarrow \mathbf{A}_{3}$.

Let $\mathbf{A}(\alpha)$ or $\mathbf{A}_{i}(\alpha)$ denote the restriction of $\mathbf{A}$ or $\mathbf{A}_{i}$ to a specific $\alpha$, for $i=1,2,3$.

TheOREM 8. ZFC $+\mathbf{A}_{2}(\alpha)$ implies that for every ordinal $\gamma<\alpha^{+}$there exists an ordinal-definable injection $f: \gamma \rightarrow \alpha$. In other words, HOD evaluates $\alpha^{+}$correctly. 
Proof. By $\mathbf{A}_{2}(\alpha)$ there exists an ordinal $\xi$ such that all ordinals less than $\gamma$ are definable in $\left\langle V_{\alpha+\xi}, \in, \eta\right\rangle_{\eta<\alpha}$. This defines an injection $f$ of $\gamma$ into a set of least size definitions in the language of that model. Of course there exists an ordinal-definable bijection between the set of formulas of that model and $\alpha$. Thus if we choose $\zeta$ such that $\alpha, \gamma$ and $\xi$ are definable in $\left\langle V_{\zeta}, \in\right\rangle$, then $f$ is also definable in $\left\langle V_{\zeta}, \in\right\rangle$.

Enayat $[\mathrm{E}]$ and Woodin $\left[\mathrm{W}_{1}\right]$ proved some consequences of $\mathbf{A}\left(\aleph_{0}\right), \mathbf{A}_{2}\left(\aleph_{0}\right)$ and $\mathbf{A}_{3}$, showing that each of these axioms implies the existence of very irregular ordinal-definable sets. With their permission we add the following information about their results.

We introduce the usual Cantor set topology in $P(\omega)$.

Theorem 9 (W. H. Woodin). ZFC $+\mathbf{A}_{2}\left(\aleph_{0}\right)$ implies the existence of uncountable sets $X \subseteq P(\omega), X \in \mathrm{OD}$, such that $X$ has no perfect subsets.

Proof. By Theorem $8, \mathbf{A}_{2}\left(\aleph_{0}\right) \Rightarrow \aleph_{1}^{\mathrm{HOD}}=\aleph_{1}$. Thus for every $\xi<\omega_{1}$, there exists an ordinal-definable injection of $\xi$ into $\omega$. Let $f_{\xi}$ be the first such injection with respect to the natural well-ordering of $\mathrm{OD}$, and let $x_{\xi}=$ $\left\{p(m, n): f_{\xi}^{-1}(m)<f_{\xi}^{-1}(n)\right\}$, where

$$
p(m, n)=\left(\begin{array}{c}
m+n+1 \\
2
\end{array}\right)+n .
$$

Then the set $X=\left\{x_{\xi}: \xi<\omega_{1}\right\}$ is ordinal-definable and $|X|=\aleph_{1}$. But $X$ has no perfect subsets since the Boundedness Theorem of descriptive set theory says that a perfect (or even analytic) set of codes of ordinals can represent only countably many ordinals.

Before stating the theorem of Enayat recall that $\mathrm{ZF}+\mathbf{A}_{3}\left(\aleph_{0}\right)$ implies the axiom $\mathbf{L}$.

Theorem 10 (A. Enayat). ZFC $+\mathbf{L}$ implies the existence of definable sets of real numbers which are not Lebesgue-measurable and do not have the property of Baire.

Proof (in outline). It is shown in [E] that $\mathbf{L}$ implies that for every set $A$ there exists a linear ordering of $A$ which is definable in terms of $A$. Hence the set $P(P(\omega))$ has a definable linear ordering. It was shown by Sierpiński $[\mathrm{S}]$ that one can define sets of reals as required in Theorem 10 in terms of any linear ordering of $P(P(\omega))$.

(Each of Theorems 9 and 10 implies a negative solution of Problem 1 of $\left[\mathrm{M}_{1}\right]$, namely that our axioms refute the Axiom of Determinacy restricted to ordinal definable subsets of ${ }^{\omega_{2}}$.)

A second theorem of Woodin $\left[\mathrm{W}_{1}\right]$ says that ZFC $+\mathbf{A}\left(\aleph_{0}\right)$ implies the existence of sets $X \subseteq{ }^{\omega} 2, X \in \mathrm{OD}$, for which the axiom of determinacy fails. 
[Adapting the proof of a well known theorem of R. M. Solovay (see [Ke, Theorem 7.3]), he shows that if all sets $X \subseteq{ }^{\omega} 2, X \in \mathrm{OD}$, were determined, then HOD would think that $\omega_{1}$ is a measurable cardinal. But this is inconsistent with $\mathbf{A}\left(\aleph_{0}\right)$ since the latter implies that there exists an injection of $\omega_{1}$ into $P(\omega)$ which belongs to HOD.]

A third theorem of Woodin $\left[\mathrm{W}_{1}\right]$ says that ZFC $+\mathbf{A}_{3}$ implies the existence of a cardinal $\alpha \leq \aleph_{2}$ and a set $X \subseteq{ }^{\alpha} 2,|X|>\alpha, X \in \mathrm{OD}$, such that $X$ has no subset lexicographically isomorphic to ${ }^{\alpha} 2$. Theorem 9 and the latter imply in particular that if $\mathbf{A}_{2}\left(\aleph_{0}\right)$ or $\mathbf{A}_{3}$ are accepted, then the class $L\left[{ }^{\mathrm{cf}(\alpha)} \alpha\right]$ in the statement $\mathbf{B}_{1}$ cannot be replaced by the class OD.

7. Some concluding remarks. 1. As mentioned in Section 1, at present there are no compelling reasons for rejecting $V=$ OD from the canonical set theory. And in spite of the fact that our axioms $\mathbf{A}_{i}(i=1,2,3)$ follow from $V=$ OD we have no arguments supporting $V=$ OD like those in Sections 2 and 6 supporting the $\mathbf{A}_{i}$ 's. Of course, if $V=\mathrm{OD}$, then $\mathbf{B}$ and $\mathbf{B}_{0}$ are equivalent to GCH. I think that the nature of the real line is so different from the nature of Ord that there is a good chance that some natural and interesting axiom will be found which will imply that some real numbers are outside of OD.

2. Concerning the concept of a canonical set theory which was introduced at the beginning of this paper, this is the set theory which a Platonist would call true. But I do not believe in the existence of a Platonic (nonphysical) reality of mathematical objects. (See $\left[\mathrm{M}_{1}, \mathrm{M}_{3}, \mathrm{M}_{4}, \mathrm{M}_{5}\right]$ for more detailed discussions of this view.) Hence I think that for the case of ZFC the concept of truth in its usual meaning does not apply. Indeed, truth is an agreement with reality, therefore it makes sense only for those mathematical theories, necessarily weaker than ZFC, which are intended to be descriptions of some physical reality. Still, a canonical set theory extending ZFC is motivated by its interesting and beautiful formal qualities, by its consequences for sets of lower ranks, by the fact that it unifies mathematics in a deep sense, and because it is a natural extension of the logic which evolved in human brains.

\section{References}

[CMP] K. Ciesielski, A. Millán and J. Pawlikowski, Uncountable $\gamma$-sets under axiom $\mathrm{CPA}_{\text {cube }}^{\text {game }}$, Fund. Math. 176 (2003), 143-155.

[CP] K. Ciesielski and J. Pawlikowski, Covering Property Axiom $\mathrm{CPA}_{\text {cube }}$ and its consequences, ibid., 63-75.

[D] F. R. Drake, Set Theory: An Introduction to Large Cardinals, North-Holland and American Elsevier, 1974. 
[E] A. Enayat, On the Leibniz-Mycielski axiom in set theory, to appear.

[FW] M. Foreman and W. H. Woodin, The generalized continuum hypothesis can fail everywhere, Ann. of Math. 133 (1991), 1-35.

[Ka] A. Kanamori, The Higher Infinite, Springer, Berlin, 1994.

$[\mathrm{Ke}] \quad$ A. S. Kechris, $A D$ and projective ordinals, in: Cabal Seminar 76-77, Lecture Notes in Math. 689, Springer, 1978, 91-132.

[L] R. Laver, On very large cardinals, in: Paul Erdős and His Mathematics, Vol. II, Budapest, 2002, 453-469.

[LS] A. Lévy and R. M. Solovay, Measurable cardinals and the continuum hypothesis, Israel J. Math. 5 (1967), 234-248.

[Ma] D. A. Martin, Descriptive set theory: projective sets, in: Handbook of Mathematical Logic, J. Barwise (ed.), North-Holland, 1991, 783-815.

[Mc] K. McAloon, Consistency results about ordinal definability, Ann. Math. Logic 2 (1971), 449-467.

[Me] T. K. Menas, Consistency results concerning supercompactness, Trans. Amer. Math. Soc. 223 (1976), 61-91.

$\left[\mathrm{M}_{1}\right] \quad$ J. Mycielski, New set-theoretic axioms derived from a lean metamathematics, J. Symbolic Logic 60 (1995), 191-198.

$\left[\mathrm{M}_{2}\right] \quad-$, Games with perfect information, in: Handbook of Game Theory, Vol. I, Chapter 3, R. J. Aumann and S. Hart (eds.), Elsevier, 1992, 41-70.

$\left[\mathrm{M}_{3}\right] \quad-$, On the tension between Tarski's nominalism and his model theory (definitions for a mathematical model of knowledge), in: Proc. Tarski's Centennial Conf. (Warszawa, 2001), to appear.

$\left[\mathrm{M}_{4}\right] \quad-$, Russell's paradox and Hilbert's (much forgotten) view of set theory, Proc. Russell's Sympos., Munich, June 2001, to appear.

$\left[\mathrm{M}_{5}\right] \quad-$, Pure Mathematics and Physical Reality, to appear.

$\left[\mathrm{M}_{6}\right] \quad$-, Two remarks on Tychonoff's product theorem, Bull. Acad. Polon. Sci. Sér. Sci. Math. Astronom. Phys. 12 (1964), 439-441.

[MS] J. Myhill and D. S. Scott, Ordinal definability, in: Axiomatic Set Theory, Proc. Sympos. Pure Math. 13, Part 1, Amer. Math. Soc., 1971, 271-278.

[N] I. Neeman, Optimal proofs of determinacy, Bull. Symbolic Logic 1 (1995), 327339.

[P] J. B. Paris, Minimal models of ZF, in: Proc. Bertrand Russell Memorial Logic Conf. (Uldum, 1971), Bertrand Russell Memorial Logic Conf. (Leeds, 1973), $327-331$.

[S] W. Sierpiński, Sur un problème conduisant à un ensemble non mesurable, Fund. Math. 10 (1927), 177-179.

$\left[\mathrm{Si}_{1}\right] \quad$ J. H. Silver, On the singular cardinals problem, in: Proc. Internat. Congress of Mathematicians (Vancouver, B.C., 1974), Vol. I, Canad. Math. Congress, Montréal, 1975, 265-268.

[Si $\left.\mathrm{S}_{2}\right] \quad$ - The independence of Kurepa's conjecture and two-cardinal conjectures in model theory, in: Axiomatic Set Theory, Proc. Sympos. Pure Math. 13, Part 1, Amer. Math. Soc., 1971, 383-390.

[So] R. M. Solovay, Strongly compact cardinals and the GCH, in: Proc. Tarski Sympos., L. Henkin et al. (eds.), Proc. Sympos. Pure Math. 25, Amer. Math. Soc., 1974, 365-372.

[St] J. R. Steel, Long games, in: Cabal Seminar 81-85, Lecture Notes in Math. 1333, Springer, 1988, 56-97.

[T] S. Todorčević, Generic absoluteness and the continuum, manuscript, May 10, 2002. 
$\left[\mathrm{W}_{1}\right] \quad$ W. H. Woodin, letters to the author, May and September 2000.

$\left[\mathrm{W}_{2}\right]$ - , The Continuum Hypothesis, Notices Amer. Math. Soc. 48 (2001), part I, 567-576, part II, 681-690; correction to part II, ibid. 49 (2002), 46.

Department of Mathematics

University of Colorado

Boulder, CO 80309-0395, U.S.A.

E-mail: jmyciel@euclid.colorado.edu

Received 22 May 2001;

in revised form 6 September 2002 and 2 January 2003 